\title{
Verde: Processing and gridding spatial data using Green's functions
}

\section{DOI: $10.21105 /$ joss.00957}

\section{Software}

- Review ct

- Repository ca

- Archive [

Submitted: 14 September 2018 Published: 11 October 2018

\section{License}

Authors of papers retain copyright and release the work under a Creative Commons Attribution $4.0 \mathrm{In}$ ternational License (CC-BY).

\section{Leonardo Uieda ${ }^{1}$}

1 Department of Earth Sciences, SOEST, University of Hawai'i at Mānoa, Honolulu, Hawaii, USA

\section{Summary}

Measurements made on the surface of the Earth are often sparse and unevenly distributed. For example, GPS displacement measurements are limited by the availability of ground stations and airborne geophysical measurements are highly sampled along flight lines but there is often a large gap between lines. Many data processing methods require data distributed on a uniform regular grid, particularly methods involving the Fourier transform or the computation of directional derivatives. Hence, the interpolation of sparse measurements onto a regular grid (known as gridding) is a prominent problem in the Earth Sciences.

Popular gridding methods include kriging, minimum curvature with tension (W. Smith \& Wessel, 1990), and bi-harmonic splines (D. T. Sandwell, 1987). The latter belongs to a group of methods often called radial basis functions and is similar to the thin-plate spline (Franke, 1982). In these methods, the data are assumed to be represented by a linear combination of Green's functions,

$$
d_{i}=\sum_{j=1}^{M} p_{j} G\left(\mathbf{x}_{i}, \mathbf{x}_{j}\right)
$$

in which $d_{i}$ is the $i$ th datum, $p_{j}$ is a scalar coefficient, $G$ is a Green's function, and $\mathbf{x}_{i}$ and $\mathbf{x}_{j}$ are the position vectors for the datum and the point defining the Green's function, respectively. Interpolation is done by estimating the $M p_{j}$ coefficients through linear leastsquares and using them to predict data values at new locations on a grid. Essentially, these methods are linear models used for prediction. As such, many of the model selection and evaluation techniques used in machine learning can be applied to griding problems as well.

Verde is a Python library for gridding spatial data using different Green's functions. It differs from the radial basis functions in scipy.interpolate by providing an API inspired by scikit-learn (Pedregosa et al., 2011). The Verde API should be familiar to scikitlearn users but is tweaked to work with spatial data, which has Cartesian or geographic coordinates and multiple data components instead of an $\mathrm{X}$ feature matrix and $\mathrm{y}$ label vector. The library also includes more specialized Green's functions (D. T. Sandwell \& Wessel, 2016), utilities for trend estimation and data decimation (which are often required prior to gridding (W. Smith \& Wessel, 1990)), and more. Some of these interpolation and data processing methods already exist in the Generic Mapping Tools (GMT) (Wessel, Smith, Scharroo, Luis, \& Wobbe, 2013), a command-line program popular in the Earth Sciences. However, there are no model selection tools in GMT and it can be difficult to separate parts of the processing that are done internally by its modules. Verde is designed to be modular, easily extended, and integrated into the scientific Python ecosystem. It can be used to implement new interpolation methods by subclassing the 
verde.base.BaseGridder class, requiring only the implementation of the new Green's function. For example, it is currently being used to develop a method for interpolation of 3-component GPS data (Uieda, Sandwell, \& Wessel, 2018).

\section{Acknowledgements}

I would like to thank collaborators Paul Wessel and David Hoese, reviewers Dom Fournier and Philippe Rivière, and editor Lindsey Heagy for helpful discussions and contributions to this project. This is SOEST contribution 10467.

\section{References}

Franke, R. (1982). Smooth interpolation of scattered data by local thin plate splines. Computers \& Mathematics with Applications, 8(4), 273-281. doi:10.1016/08981221(82)90009-8

Pedregosa, F., Varoquaux, G., Gramfort, A., Michel, V., Thirion, B., Grisel, O., Blondel, M., et al. (2011). Scikit-learn: Machine Learning in Python. Journal of Machine Learning Research, 12(Oct), 2825-2830.

Sandwell, D. T. (1987). Biharmonic spline interpolation of GEOS-3 and SEASAT altimeter data. Geophysical Research Letters, 14(2), 139-142. doi:10.1029/GL014i002p00139

Sandwell, D. T., \& Wessel, P. (2016). Interpolation of 2-D vector data using constraints from elasticity. Geophysical Research Letters, 43(20), 2016GL070340. doi:10.1002/2016GL070340

Smith, W., \& Wessel, P. (1990). Gridding with continuous curvature splines in tension. Geophysics, 55(3), 293-305. doi:10.1190/1.1442837

Uieda, L., Sandwell, D., \& Wessel, P. (2018). Presentation: Joint Interpolation of 3component GPS Velocities Constrained by Elasticity. figshare. doi:10.6084/m9.figshare.6387467

Wessel, P., Smith, W. H. F., Scharroo, R., Luis, J., \& Wobbe, F. (2013). Generic Mapping Tools: Improved Version Released. Eos, Transactions American Geophysical Union, 94(45), 409-410. doi:10.1002/2013E0450001 